\title{
A Case Series of Severe Dengue with Neurological Presentation in Children from a Colombian Hyperendemic Area
}

\author{
Jaime E. Castellanos $\mathbb{D}^{1},{ }^{1}$ Paula Esteban, ${ }^{2}$ Juanita Panqueba-Salgado, ${ }^{2}$ \\ Daniela Benavides-del-Castillo, ${ }^{2}$ Valentina Pastrana, ${ }^{2}$ Gladys Acosta, ${ }^{3}$ Doris Salgado (D), \\ Carlos F. Narvaez $(\mathbb{D})^{3}$ Sigrid Camacho-Ortega $\mathbb{D}^{1},{ }^{1}$ Eliana Calvo $\mathbb{D}{ }^{1}$ \\ and Myriam L. Velandia-Romero $\mathbb{D}^{1}$ \\ ${ }^{1}$ Grupo de Virología, Universidad El Bosque, Bogotá, Colombia \\ ${ }^{2}$ Facultad de Medicina, Universidad El Bosque, Bogotá, Colombia \\ ${ }^{3}$ Grupo de Parasitología y Medicina Tropical, Universidad Surcolombiana, Neiva, Colombia
}

Correspondence should be addressed to Jaime E. Castellanos; castellanosjaime@unbosque.edu.co

Received 13 October 2020; Revised 24 April 2021; Accepted 24 May 2021; Published 1 June 2021

Academic Editor: Thomas R. Chauncey

Copyright (C) 2021 Jaime E. Castellanos et al. This is an open access article distributed under the Creative Commons Attribution License, which permits unrestricted use, distribution, and reproduction in any medium, provided the original work is properly cited.

\begin{abstract}
Dengue transmission is sustained in Colombia with increasing prevalence mainly in children. This work aimed to describe a case series of children diagnosed with dengue presenting neurological disease in Huila Province of Colombia. Eleven pediatric febrile patients confirmed for dengue disease and presenting neurological signs were studied in the University Hospital of Neiva, Huila Province. Clinical and laboratory findings, CSF cytochemical analysis, neurology images, and serology and molecular studies were performed. Viral RNA was detected in all patients' sera by RT-PCR. Nine out of 11 were primary infections. Tonic-clonic seizures (73\%), consciousness alterations (27\%), irritability (27\%), and ataxia (18\%) were the most frequent neurological signs. None of the patients had plasma leakage, hypovolemic shock, or liver disease, confirming the encephalitis diagnosis. Diagnostic images did not show abnormal findings, but neither bacterial nor fungal infections were detected in CSF analysis. All patients survived without sequelae except for one patient that presented ataxia for months. In conclusion, we described a group of children with neurological signs during severe dengue disease as the main finding, indicating the importance to including dengue as a differential diagnosis in neurological patients from endemic areas.
\end{abstract}

\section{Introduction}

The world estimate of dengue infections is around 390 million people among symptomatic and asymptomatic cases in endemic countries such as those of Latin America and the Asian, Southeast, and Pacific Islands [1]. Nearly 65\% of Colombian municipalities reported constant Aedes infestation [2] and dengue incidence increase year after year mainly associated with abnormal environmental sanitary conditions, broad circulation of the four serotypes, and high mosquito infestation [3, 4]. In America, most of the cases occur in individuals under 15 years of age [5].

The largest dengue occurred in Latin America was reported in 2019 with more than three million cases [6] reaching an incidence rate of 80 cases per 100,000 inhabitants.

The current disease classification propose is to identify dengue following clinical signs and symptoms to establish the most accurate diagnosis and recognize early cases that could become severe or fatal [7]. Accordingly, dengue warning signs reflecting endothelial dysfunction that should be revised are abdominal tenderness, vomit, edema, mucosal bleeding, hepatomegaly, irritability, or hypotension. In those presumptive dengue cases occurring with plasma leakage signs (pleural effusion and pericarditis) or respiratory distress, severe bleeding, or affectation of organs such as the liver, brain, or kidney, a diagnostic of severe dengue should be corroborated [8]. 
Even although neurological manifestations were reported since 1976 in Thailand as "dengue-associated acute encephalopathy" [9], from these years until today, the incidence of neurologic manifestations during dengue disease has increased considerably [10]. Similar cases have been reported from the Asian, Southeast, and Pacific Islands of America [11, 12]. Today, some hypotheses proposes that DENV could also be neurotropic as other flaviviruses supported by the frequent finding of specific IgM or viral RNA in the cerebrospinal fluid (CSF) $[13,14]$ that could confirm CNS infection and direct brain injury. This finding allows the differentiation of those circulating viruses and antibodies that could enter to the CNS due to impairment of the blood-brain barrier (BBB).

Many kinds of pathologies have been reported, from cerebral edema and hemorrhage to perivascular lymphocyte infiltrates and demyelination foci. These anomalies could be explained by i) BBB alterations induced by TNF-alpha and IL-6 activity involved in endothelial junction disruption favoring virus entry to the CNS, ii) DENV infection to endothelial cells leading to virion pass through to brain parenchyma $[15,16]$, and iii) endothelial cell injury by glycocalyx remodeling induced by NS1. Nervous system DENV replication has been observed in the olivary nucleus and cerebellum as well as tissue macrophages DENV positive, indicating some neurotropism [17].

It has been reported that $47 \%$ of adult meningitis and encephalitis cases in a Brazilian endemic area were explained by acute DENV infection occurring during the first week of fever onset [18]. During that outbreak, the disorder did appear as encephalitis with behavioral disorders, seizures, and paresis with no evidence of liver failure and confirming serum or CSF IgM serology or altered neuroimages showing brain changes. Other cases with unspecific neurological signs could appear as a consequence of metabolic alterations or prolonged shock, brain hypoxia, cerebral edema, or liver or kidney failure that explain the headache, retro-orbital pain, insomnia, restlessness, and mood changes appearing in patients [19]. The Colombian study reported by Mendez and Gonzalez [20] described 46 out of 168 severe dengue child patients presenting encephalopathy and 3 with encephalitis diagnostic. Dengue encephalitis is considered when febrile patients in endemic areas, having one of these signs or symptoms, headache, seizures, consciousness alterations, and behavioral changes with no hepatic failure or bleeding and positive for DENV serology or molecular test in serum or CSF with altered brain images in tomography or resonance. The purpose of this article is to present and describe the clinical, laboratory, and diagnostic characteristics of eleven children diagnosed as dengue cases, which presented neurological manifestations, cases occurring during regular endemic circulation in the Colombian province of Huila in 2011. DENV spreading in Latin-American countries and inside of them has been explained by air traffic between cities, urban population size, and human development index, which predict positively the outbreaks in those endemic cities like Neiva studied here (Allicock).

\section{Cases Presentation}

The University Hospital Hernando Moncaleano is the largest health setting in Neiva, the capital city of Huila Province, located at 42 meters above sea level with a mean temperature of $28^{\circ} \mathrm{C}$ where it has been reported frequent dengue outbreaks. The Hospital Institutional Review Board approved the study and parents or guardians signed the consent form during the stay. We collected the clinical and laboratory information of those febrile patients with one of dengue symptoms such as rash, headache, retroorbital pain, myalgia, and arthralgia associated with any neurological signs such as seizures, abnormal movements, acute flaccid palsy, lethargy, meningeal signs, or consciousness alteration with no shock evidence, which were diagnosed as severe dengue following the recommendation of the WHO. Clinical records from the pediatric emergency department were revised from March to October 2011 and those patients with neurological signs and another microorganism associated were excluded. One specific designed clinical report form was filled for each case, and complete information of laboratory tests (blood cell counts, coagulation tests, liver, and kidney function markers), diagnostic images (tomography and chest $\mathrm{Rx}$ ), and CSF chemical and microbiological analysis was collected; however, other viral pathogens were not evaluated. Sera from the enrolled cases were processed for serum IgM and capture IgG detection by ELISA and for viral RNA detection by RT-PCR on sera following the protocol described previously [21].

During the study period, 34 patients with fever and neurological manifestations were examined, but three were excluded (one by mental retardation and two with psychiatric disorders); the remaining 31 patients were investigated for dengue by serology or sera RT-PCR, and eleven (35.5\%) tested positive for any of them (two girls and nine boys). All of them, finally, were discharged with severe dengue diagnostic or viral encephalitis, despite all of them having positive dengue IgM or IgG test in serum. All but one patient had laboratory analysis, although this one had serology and RT-PCR results. Most of the patients (73\%) presented seizure disorder or convulsions. In Table 1, the complete data of patients are shown. Although all patients were IgM positive, only two were IgG positive (secondary infection). All sera were positive for RT-PCR for DENV, but only three of them allowed serotyping (DENV-1).

Clinically, eight patients presented tonic-clonic seizures although only $2 / 11$ reported headache and three developed consciousness alteration or irritability, two had ataxia symptoms, and one developed unilateral paresis. Other signs found were vomiting (5), hepatomegaly (5), cough (5), abdominal pain (3), asthenia (3), and epistaxis (2). None of the patients presented plasma leakage signs such as edema or pleural effusion, nor altered transaminase values. There was brain tomography in eight patients and one magnetic resonance, all showing no alterations. Additionally, chest $\mathrm{Rx}$ from three patients was normal. Despite the severity, there was no fatal case, and all of them, but one, recovered without sequelae. 


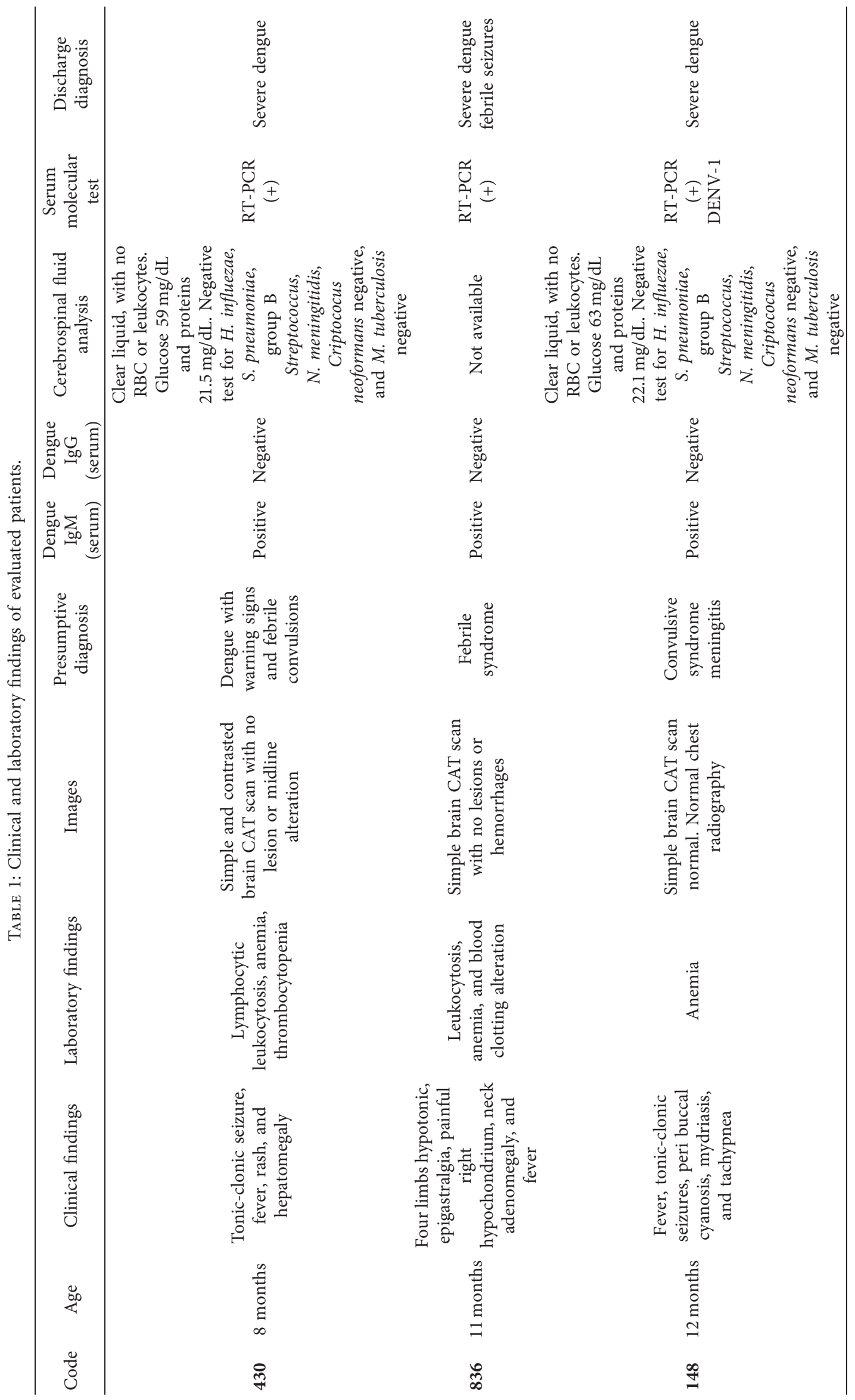




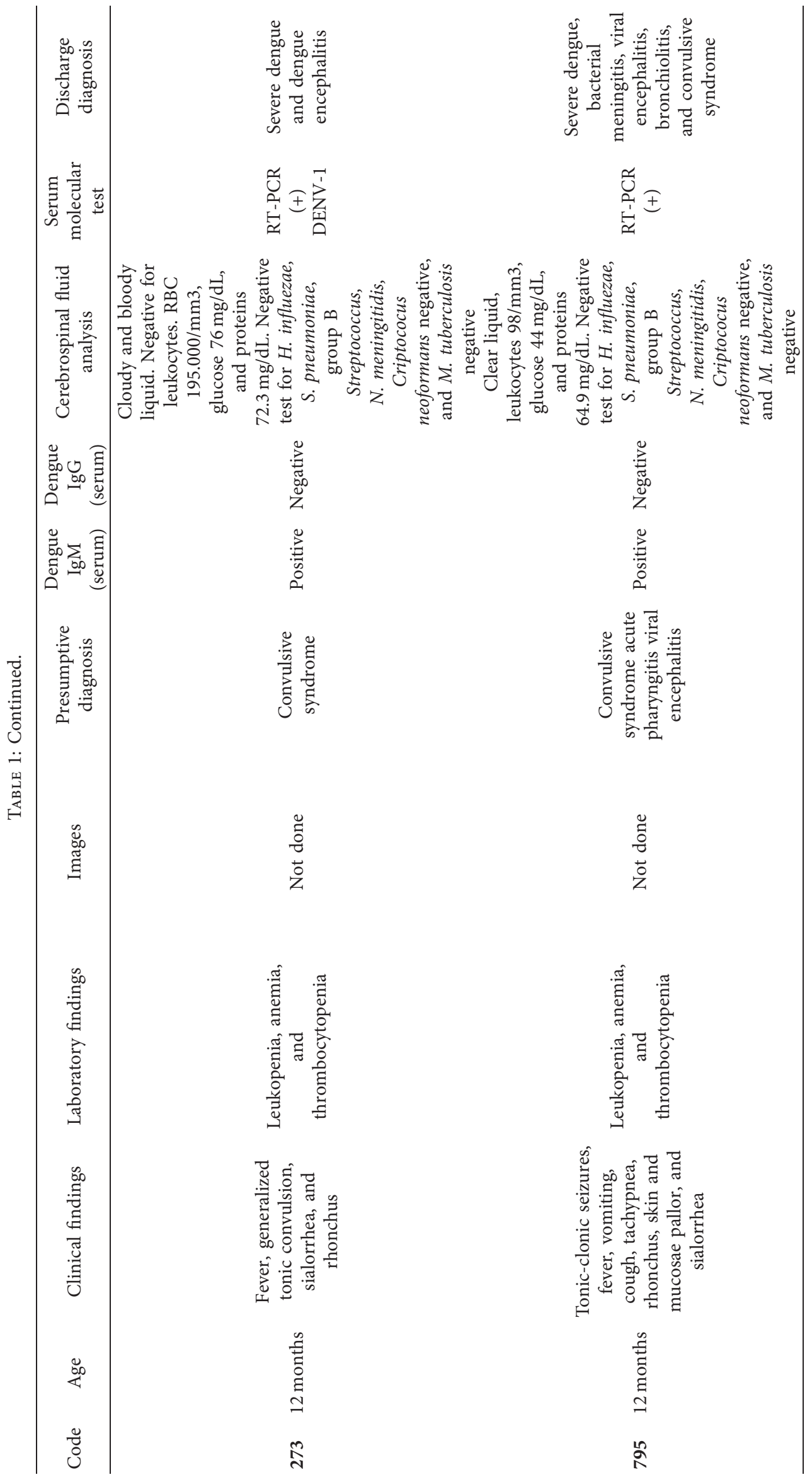




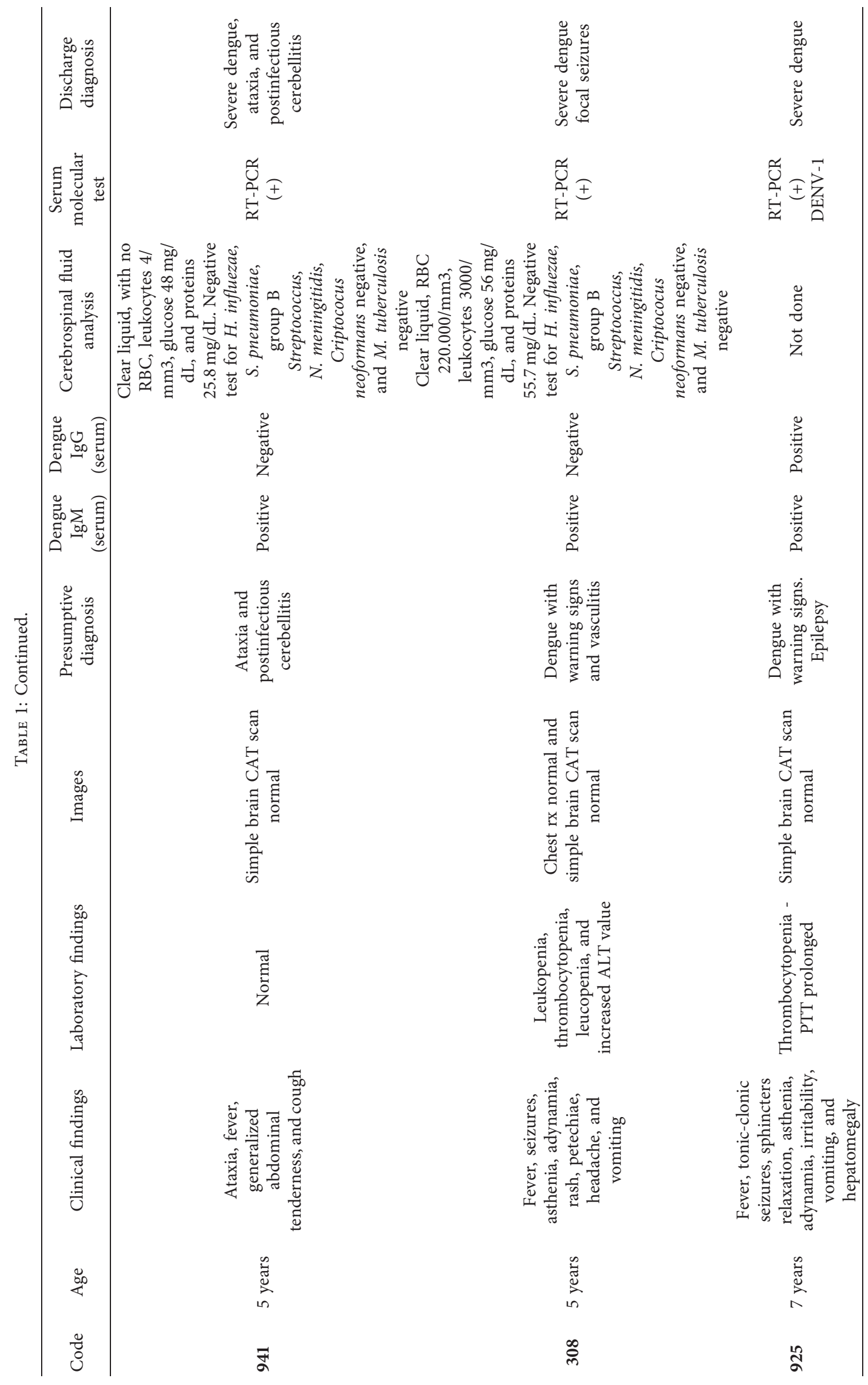




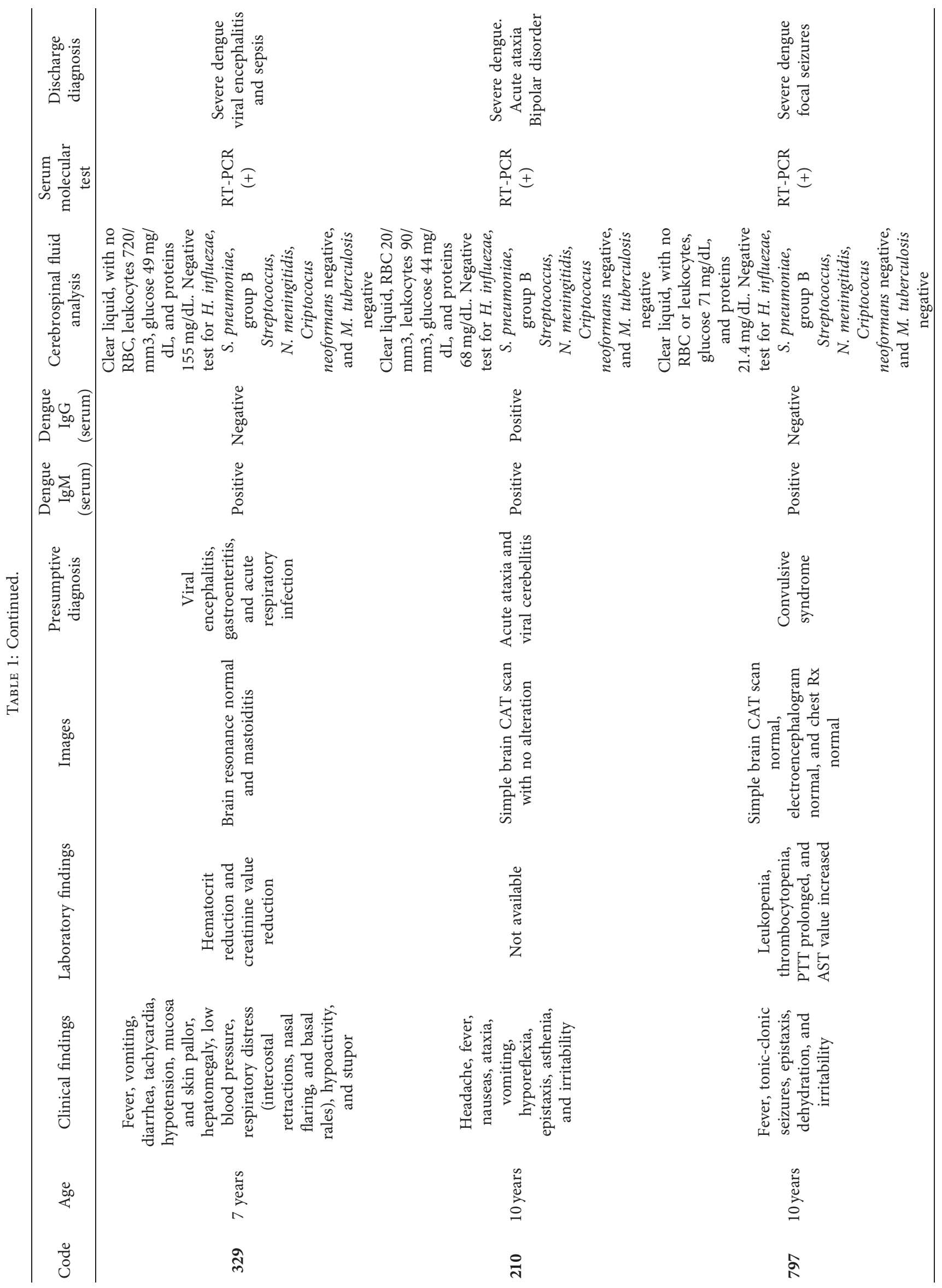




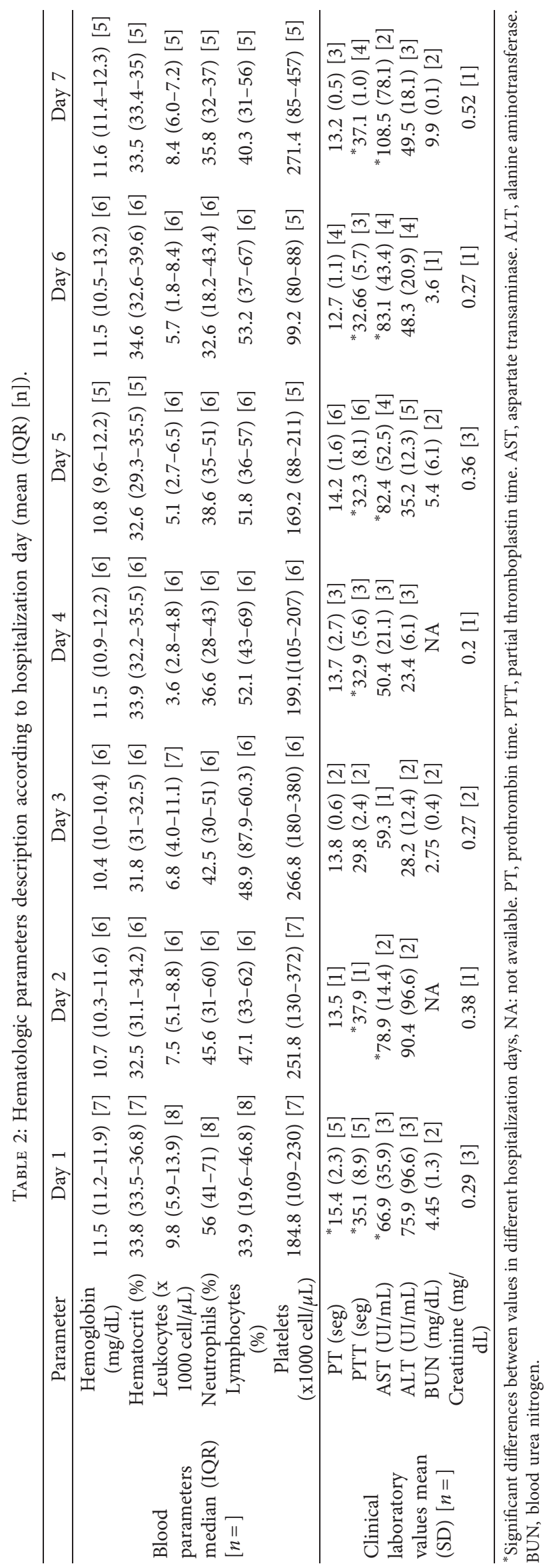


The hematimetric analysis indicated anemia and low hematocrit in all patients (median 33.5\%, IQR 31.8-34.6\%) since the child normal value is $36-42 \%$. A mild leukocytosis was observed at day one and a decrease until day four when leukopenia was evident. Six patients developed thrombocytopenia with the day 6 value being the lowest (median 99.200 platelets $/ \mathrm{mm}^{3}$ ). The coagulation prothrombin test only showed a mean of $15.4 \mathrm{~s}$; it means a prolongation value at the first hospitalization day. However, the partial thromboplastin time (PTT) was prolonged during six hospitalization days with values between 32.3 and $35.1 \mathrm{~s}$ (Table 2). Transaminase levels used to evaluate the hepatic function and AST showed a slight increase during the last three hospitalization days (fifth to the seventh day), but the values were close to those reference levels while ALT did not show changed levels. There were no changes in renal function markers in any of the patients.

\section{Discussion}

Colombia has seen an increase in the dengue cases number in the last decade with higher severity and mortality $[4,5]$, forcing medical personnel and health authorities to strengthen capacity to recognize and differentiate classical dengue signs and symptoms from the atypical and severe presentation. After the redefinition of diagnostic and severity criteria by the WHO [22], severe dengue is defined when patients present organ damage, associated signs (liver, brain, and kidney), or hypovolemic shock or massive bleeding. This system facilitates the early recognition of the most severe cases by reducing the mortality numbers.

This study presented eleven severe dengue cases in children between eight months to 10 years of age, living in the Colombian province Huila, a hyperendemic area that reported two-fifths of severe dengue cases registered from 2000 to 2011 [5]. All patients presented brain compromise; nine were diagnosed as not only encephalitis (81.8\%) following the criteria described previously [23], such as fever, sensory disturbances, seizures, or focal neurological signs, but also positive tests (IgM or NS1) or RT-PCR in serum or CSF and negative for others encephalitis causes. This presentation was the most common compromise in this series, characterized by seizures in six patients (tonic-clonic convulsions) as has been previously reported in children [24]. There was one severe sensory impairment case, and another presented with severe headache, which also is frequently described in dengue encephalitis [25]. Less than half of the described patients develop classical dengue signs such as vomiting, hepatomegaly, and exanthem, indicating the neurological cases do not seem to have dengue disease and that nervous signs could appear alone leading to registration of severe cases and possibly in the delay of the establishment of adequate treatment [23]. Two patients were diagnosed as cerebellitis, characterized by nystagmus, dysarthria, or ataxia, which also has been reported previously [26]. Fortunately, there were no fatal cases in this patient group; on the contrary, outcomes and recovery were satisfactory.

It is recommended that, in DENV circulation areas, those patients with fever and neurologic signs should be investigated for dengue disease, ensuring laboratory confirmation using both serology and molecular tests that, together, could offer high sensitivity [26]. Here, we used both IgM and IgG serology in addition to RT-PCR and found all serum samples positive, even serotyping DENV-1 in three patients, which is not the most reported associated with neuroinfection [27]. The finding of nine sera negative for IgG is very odd because most of the severe dengue cases are during secondary infections with a different serotype, where the antibodies are not capable of the second virus neutralization but induce an enhanced infection and an aberrant immune response. Therefore, severe dengue with a primary compromise of an organ in primoinfection could suggest a neurotropic strain or a host characteristic that determined the neuroinfection in this child group.

All CSF samples were negative for bacteria or fungi, but five out of nine had lymphocyte pleocytosis, an uncommon sign in dengue encephalitis; since most of the cases do not develop CSF anomalies [24], even three-quarters of dengue encephalitis or meningitis patients showed normal values in CSF analysis [27]. Despite previous studies reported that brain tissue damage is detectable by tomography images, the children described in this work did not present anomalies. Although it is preferable to use magnetic resonance for brain evaluation because of the accuracy of this technique, regularly imaging abnormalities during dengue encephalitis are not specific [28] and are still poorly defined [29, 30], although ischemia or hemorrhage must be sought [31]. On the other hand, we found normal leukocyte counts instead of the classical leukopenia of dengue with warning signs or severe dengue, which pose a difficulty to early diagnosis, although there was thrombocytopenia only at day six of hospitalization with no changes in ALT and AST transaminases as reported previously. Recently, 11/36 patients from a Brazilian endemic area were diagnosed with neurologic manifestations and confirmed for arbovirus infection using CSF or sera [32] (5 DENV and $6 \mathrm{CHIKV),} \mathrm{posing} \mathrm{an} \mathrm{additional}$ warning about involvement of these viruses in severe disease presentation in Latin-American countries where there is cocirculation.

\section{Conclusions}

This child group were diagnosed as severe dengue with central nervous system manifestations without shock, typical signs, or hemorrhage. Therefore, we should recommend that, in endemic dengue areas, health professionals should include infections by this virus in those patients with fever and neurological manifestations as part of the primary diagnostic and must be vigilant for neurological signs that could appear after acute dengue disease.

\section{Limitations}

This study was retrospective and is limited by its observational nature. Although we enrolled almost all those children with severe dengue diagnosis in this hospital, detailed clinical and laboratory information was obtained from these 
reported patients after an in-depth search of data in the medical records.

\section{Data Availability}

The dataset supporting the conclusions of this article is available in the http://www.researchgate.net repository, DOI: 10.13140/RG.2.2.32618.54728 and DOI: 10.13140/ RG.2.2.15841.33128.

\section{Ethical Approval}

This study was approved by the Institutional Review Board of Hospital Hernando Moncaleano Perdomo and was revised and approved by the Ethics Institutional Review Board of Universidad El Bosque (Minute 007-2014).

\section{Consent}

Parents signed an informed consent form. Names and characteristics of each patient were blinded and coded to ensure their privacy.

\section{Conflicts of Interest}

The authors declare that they have no conflicts of interest.

\section{Authors' Contributions}

Jaime E. Castellanos was the principal investigator and analyzed data, wrote the manuscript, and edited the manuscript. Paula Esteban, Juanita Panqueba-Salgado, Daniela Benavides-del-Castillo, and Valentina Pastrana collected clinical and laboratory information, analyzed data, and wrote the manuscript draft. Gladys Acosta performed clinical evaluation, brain images analysis, laboratory data analysis, sample collection, and manuscript editing. Doris Salgado performed clinical evaluation, brain image analysis, sample collection, and manuscript writing and editing. Carlos F. Narvaez performed serology tests, laboratory data analysis, and manuscript editing. Sigrid Camacho-Ortega carried out serum sample processing and molecular diagnosis and wrote the manuscript draft. Eliana Calvo carried out serum sample processing and dengue molecular diagnosis and edited the manuscript. Myriam Velandia-Romero was the co-Investigator of data analysis and carried out manuscript revision.

\section{Acknowledgments}

This project was partially funded by COLCIENCIAS (Red de investigación multidisciplinaria para la prevención y control de enfermedades trasmitidas por vectores Red ETV, Grant 360-2011) and Universidad El Bosque (Vicerrectoria de Investigaciones). The authors thank Miguel Otero Cadena, Vice Principal of Research of Universidad El Bosque, for the invaluable and constant support during the project development.

\section{References}

[1] S. Bhatt, P. W. Gething, O. J. Brady et al., "The global distribution and burden of dengue," Nature, vol. 496, no. 7446, pp. 504-507, 2013.

[2] R. Pérez-Castro, J. E. Castellanos, V. A. Olano et al., "Detection of all four dengue serotypes in Aedes aegypti female mosquitoes collected in a rural area in Colombia," Memórias Do Instituto Oswaldo Cruz, vol. 111, no. 4, pp. 233-240, 2016.

[3] J. C. Padilla, F. E. Lizarazo, O. L. Murillo, F. A. Mendigaña, E. Pachón, and M. J. Vera, "Epidemiología de las principales enfermedades transmitidas por vectores en Colombia, 19902016," Biomédica, vol. 37, pp. 27-40, 2017.

[4] J. C. Castrillón, J. C. Castaño, and S. Urcuqui, "Dengue en Colombia: diez años de evolución," Revista chilena de infectología, vol. 32, no. 2, pp. 142-149, 2015.

[5] L. A. Villar, D. P. Rojas, S. Besada-Lombana, and E. Sarti, "Epidemiological trends of dengue disease in Colombia (2000-2011): a systematic review," PLOS Neglected Tropical Diseases, vol. 9, no. 3, Article ID e0003499, 2015.

[6] H. Gutierrez-Barbosa, S. Medina-Moreno, J. C. Zapata, and J. V. Chua, "Dengue infections in Colombia: Epidemiological trends of a hyperendemic Country," Tropical Medicine and Infectious Disease, vol. 5, no. 4, p. 156, 2020.

[7] D. M. Salgado, M. R. Vega, C. A. Panqueba, C. F. Narváez, and J. A. Rodríguez, "Análisis descriptivo del compromiso de órganos en niños con dengue grave en Neiva, Colombia," Revista de la Facultad de Medicina, vol. 65, no. 4, pp. 565-570, 2017.

[8] D. M. Salgado, J. A. Rodríguez, M. Garzón et al., "Caracterización Clínica y Epidemiológica de Dengue Hemorrágico en Neiva, Colombia, 2004," Revista de Salud Pública, vol. 9, no. 1, pp. 53-63, 2007.

[9] L. K. Kho, W. H. Sumarmo, H. Wulur, E. C. Jahja, and D. J Gubler, "Dengue hemorrhagic fever accompanied by encephalopathy in Jakarta," The Southeast Asian Journal of Tropical Medicine and Public Health, vol. 12, pp. 83-86, 1981.

[10] L. Muñoz, M. Garcia, E. Gordon-Lipkin, B. Parra, and C. Pardo, "Emerging viral infections and their Impact on the global burden of neurological disease," Seminars in Neurology, vol. 38, no. 02, pp. 163-175, 2018.

[11] J. M. K. Murthy, "Neurological complication of dengue infection," Neurology India, vol. 58, no. 4, pp. 581-584, 2010.

[12] R. B. Domingues, G. W. Kuster, F. L. Onuki-Castro, V. A. Souza, J. E. Levi, and C. S. Pannuti, "Involvement of the central nervous system in patients with dengue virus infection," Journal of the Neurological Sciences, vol. 267, no. 1-2, pp. 36-40, 2008.

[13] T. Solomon, N. M. Dung, D. W. Vaughn et al., "Neurological manifestations of dengue infection," The Lancet, vol. 355, no. 9209, pp. 1053-1059, 2000.

[14] M. M. Ngwe Tun, R. Muthugala, T. Nabeshima et al., "Complete genome analysis and characterization of neurotropic dengue virus 2 cosmopolitan genotype isolated from the cerebrospinal fluid of encephalitis patients," PLoS One, vol. 15, no. 6, Article ID e0234508, 2020.

[15] M. L. Velandia-Romero, O. Acosta-Losada, and J. E. Castellanos, "In vivo infection by a neuroinvasive neurovirulent dengue virus," Journal of NeuroVirology, vol. 18, no. 5, pp. 374-387, 2012.

[16] M.-A. Calderón-Peláez, M. L. Velandia-Romero, L. Y. Bastidas-Legarda, E. O. Beltrán, S. J. Camacho-Ortega, and J. E. Castellanos, "Dengue virus infection of blood-brain barrier cells: Consequences of severe disease," Frontiers in Microbiology, vol. 10, p. 1435, 2019. 
[17] A. Varatharaj, "Encephalitis in the clinical spectrum of dengue infection," Neurology India, vol. 58, no. 4, pp. 585-591, 2010.

[18] C. N. Soares, M. J. Cabral-Castro, J. M. Peralta, M. R. G. De Freitas, M. Zalis, and M. Puccioni-Sohler, "Review of the etiologies of viral meningitis and encephalitis in a dengue endemic region," Journal of the Neurological Sciences, vol. 303, no. 1-2, pp. 75-79, 2011.

[19] F. J. Carod-Artal, O. Wichmann, J. Farrar, and J. Gascón, "Neurological complications of dengue virus infection," The Lancet Neurology, vol. 12, no. 9, pp. 906-919, 2013.

[20] A. Méndez and G. González, "Manifestaciones clínicas inusuales del dengue hemorrágico en niños," Biomédica, vol. 26, no. 1, pp. 61-70, 2006.

[21] J. E. Castellanos, C. Coronel-Ruiz, S Parra-Alvarez et al., "Description of high rates of unapparent and simultaneous multiple dengue virus infection in a Colombian jungle settlement," Tropical Biomedicine, vol. 33, no. 2, pp. 375-382, 2016.

[22] J. Barniol, R. Gaczkowski, E. V. Barbato et al., "Usefulness and applicability of the revised dengue case classification by disease: multi-centre study in 18 countries," BMC Infectious Diseases, vol. 11, no. 1, p. 106, 2011.

[23] S. Cristiane and P.-S. Marzia, "Diagnosis criteria of dengue encephalitis," Arquivos de Neuro-Psiquiatria, vol. 72, no. 3, p. $263,2014$.

[24] G. Angibaud, J. Luaute, M. Laille, and C. Gaultier, "Brain involvement in Dengue fever," Journal of Clinical Neuroscience, vol. 8, no. 1, pp. 63-65, 2001.

[25] M. Puccioni-Sohler, C. Rosadas, and M. J. Cabral-Castro, "Neurological complications in dengue infection: a review for clinical practice," Arquivos de Neuro-Psiquiatria, vol. 71, no. 9B, pp. 667-671, 2013.

[26] P. N. Weeratunga, M. Chandragomi Caldera, I. K. Gooneratne, R. Gamage, and P. Perera, "Neurological manifestations of dengue: a cross sectional study," Travel Medicine and Infectious Disease, vol. 12, no. 2, pp. 189-193, 2014.

[27] C. N. Soares, M. J. Cabral-Castro, J. M. Peralta, M. R. G. Freitas, and M. Puccioni-Sohler, "Meningitis determined by oligosymptomatic dengue virus type 3 infection: report of a case," International Journal of Infectious Diseases, vol. 14, no. 2, pp. e150-e152, 2010.

[28] R. K. Garg, I. Rizvi, R. Ingole et al., "Cortical laminar necrosis in dengue encephalitis-a case report," BMC Neurology, vol. 17, no. 1, p. 79, 2017.

[29] T. Mathew, S. Badachi, G. Sarma, and R. Nadig, "Dot sign in dengue encephalitis," Annals of Indian Academy of Neurology, vol. 18, pp. 77-79, 2014.

[30] F. J. Carod Artal, "Complicaciones neurológicas asociadas a la infección por el virus del dengue," Revista de Neurología, vol. 69, no. 03, pp. 113-122, 2019.

[31] J. Castellanos, J. Bello, and M. Velandia-Romero, "Manifestaciones neurológicas durante la infección por el virus del dengue," Infectio, vol. 18, no. 4, pp. 167-176, 2014.

[32] C. d. S. Mello, M. J. Cabral-Castro, L. C. Silva de Faria, J. M. Peralta, and M. Puccioni-Sohler, "Dengue and chikungunya infection in neurologic disorders from endemic areas in Brazil," Neurology: Clinical Practice, vol. 10, no. 6, pp. 497-502, 2020. 